\title{
Some Properties of the Strong Primitivity of Nonnegative Tensors
}

\author{
Lihua You (D), Yafei Chen, and Pingzhi Yuan (D) \\ School of Mathematical Sciences, South China Normal University, Guangzhou 510631, China \\ Correspondence should be addressed to Lihua You; ylhua@scnu.edu.cn and Pingzhi Yuan; yuanpz@scnu.edu.cn
}

Received 25 January 2018; Accepted 3 July 2018; Published 7 August 2018

Academic Editor: Ali R. Ashrafi

Copyright (C) 2018 Lihua You et al. This is an open access article distributed under the Creative Commons Attribution License, which permits unrestricted use, distribution, and reproduction in any medium, provided the original work is properly cited.

\begin{abstract}
We show that an order $m$ dimension 2 tensor is primitive if and only if its majorization matrix is primitive, and then we obtain the characterization of order $m$ dimension 2 strongly primitive tensors and the bound of the strongly primitive degree. Furthermore, we study the properties of strongly primitive tensors with $n \geq 3$ and propose some problems for further research.
\end{abstract}

\section{Introduction}

In recent years, the study of tensors and the spectra of tensors (and hypergraphs) with their various applications has attracted extensive attention and interest, since the work of $\mathrm{L}$. Qi ([1]) and L.H. Lim ([2]) in 2005.

As is in [1], an order $m$ dimension $n$ tensor $\mathbb{A}=$ $\left(a_{i_{1} i_{2} \ldots i_{m}}\right)_{1 \leq i_{j} \leq n(j=1, \ldots, m)}$ over the complex field $\mathbb{C}$ is a multidimensional array with all entries

$$
a_{i_{1} i_{2} \cdots i_{m}} \in \mathbb{C}\left(i_{1}, \ldots, i_{m} \in[n]=\{1, \ldots, n\}\right) .
$$

A tensor $\mathbb{A}=\left(a_{i_{1} i_{2} \ldots i_{m}}\right)$ is called a nonnegative tensor if all of its entries $a_{i_{1} i_{2} \ldots i_{m}}$ are nonnegative. Clearly, adjacency tensors and signless Laplacian tensors are nonnegative.

In the theory of nonnegative matrices, the notion of primitivity plays an important role in the convergence of the Collatz method. For a nonnegative matrix $A$, the following are equivalent [3]:

(1) Let $\rho(A)$ be the spectral radius of $A$. Then $A$ is irreducible and $\rho(A)$ is greater than any other eigenvalue in modulus.

(2) The only $A$-invariant nonempty subset of the boundary of the positive cone is $\{0\}$.

(3) There exists a natural number $r$ such that $A^{r}$ is positive.

Matrices which satisfy any of the above conditions are called primitive. The least such $r$ such that $A^{r}$ is positive is called the primitive exponent (or simply, exponent) of $A$ and is denoted by $\exp (A)$.
In [4], K.C. Chang et al. defined the primitivity of nonnegative tensors (as Definition 1), extended the theory of nonnegative matrices to nonnegative tensors, and proved the convergence of the NQZ method which is an extension of the Collatz method and can be used to find the largest eigenvalue of any nonnegative irreducible tensor.

Definition 1 (see [4]). Let $\mathbb{A}$ be a nonnegative tensor with order $m$ and dimension $n, x=\left(x_{1}, x_{2}, \ldots, x_{n}\right)^{T} \in \mathbb{R}^{n}$ a vector, and $x^{[r]}=\left(x_{1}^{r}, x_{2}^{r}, \ldots, x_{n}^{r}\right)^{T}$. Define the map $T_{\mathbb{A}}$ from $\mathbb{R}^{n}$ to $\mathbb{R}^{n}$ as $T_{\mathbb{A}}(x)=\left(\mathbb{A} x^{m-1}\right)^{[1 /(m-1)]}$. If there exists some positive integer $r$ such that $T_{\mathbb{A}}^{r}(x)>0$ for all nonnegative nonzero vectors $x \in \mathbb{R}^{n}$, then $\mathbb{A}$ is called primitive and the smallest such integer $r$ is called the primitive degree of $\mathbb{A}$, denoted by $\gamma(\mathbb{A})$.

As in [1], let $\mathbb{A}=\left(a_{i_{1} i_{2} \ldots i_{m}}\right)_{1 \leq i_{j} \leq n(j=1, \ldots, m)}$ be an order $m$ dimension $n$ tensor over the complex field $\mathbb{C}, x=\left(x_{1}\right.$, $\left.\ldots, x_{n}\right)^{T} \in \mathbb{C}_{n}, x^{[r]}=\left(x_{1}^{r}, x_{2}^{r}, \ldots, x_{n}^{r}\right)$, and $\mathbb{A} x^{m-1}$ be a vector $\in \mathbb{C}_{n}$ whose $i$ th component is defined as follows:

$$
\left(A x^{m-1}\right)_{i}=\sum_{i_{2}, \ldots, i_{m}=1}^{n} a_{i i_{2} \cdots i_{m}} x_{i_{2}} \cdots x_{i_{m}} .
$$

Then a number $\lambda \in \mathbb{C}$ is called an eigenvalue of $\mathbb{A}$ if there exists a nonzero vector $x \in \mathbb{C}_{n}$ such that

$$
\mathbb{A} x^{m-1}=\lambda x^{[m-1]} .
$$


Recently, Shao [5] defined the general product of two ndimensional tensors as follows, and one of the applications of the tensor product is that $\mathbb{A} x^{m-1}$ can be simply written as A $\cdot x$.

Definition 2 (see [5]). Let $\mathbb{A}$ (and $\mathbb{B}$ ) be an order $m \geq 2$ (and $k \geq 1$ ), dimension $n$ tensor, respectively. Define the general product $\mathbb{A} \cdot \mathbb{B}$ (sometimes simplified as $\mathbb{A} \mathbb{B})$, to be the following tensor $\mathbb{D}$ of order $(m-1)(k-1)+1$ and dimension $n$ :

$$
\begin{aligned}
d_{i \alpha_{1} \cdots \alpha_{m-1}}=\sum_{i_{2}, \ldots, i_{m}=1}^{n} a_{i i_{2} \cdots i_{m}} b_{i_{2} \alpha_{1}} \cdots b_{i_{m} \alpha_{m-1}} & \\
& \quad\left(i \in[n], \alpha_{1}, \ldots, \alpha_{m-1} \in[n]^{k-1}\right) .
\end{aligned}
$$

The tensor product is a generalization of the usual matrix product and satisfies a very useful property: the associative law ([5], Theorem 1.1). By the associative law, we can define $\mathbb{A}^{k}$ as the product of $k$ many tensors $A$.

With the general product, when $k=1$ and $\mathbb{B}=x=$ $\left(x_{1}, \ldots, x_{n}\right)^{T} \in \mathbb{C}^{n}$ is a vector of dimension $n$, then $\mathbb{A} \cdot \mathbb{B}=\mathbb{A} \cdot x$ is still a vector of dimension $n$, and for any $i \in[n],(\mathbb{A} \cdot \mathbb{B})_{i}=$ $(\mathbb{A} \cdot x)_{i}=\sum_{i_{2}, \ldots, i_{m}=1}^{n} a_{i i_{2} \ldots i_{m}} x_{i_{2}} \ldots x_{i_{m}}=\left(\mathbb{A} x^{m-1}\right)_{i}$, where $\mathbb{A} x^{m-1}$ is defined in (2). Thus we have $\mathbb{A} x^{m-1}=\mathbb{A} \cdot x$.

In order to study eigenvalue, Pearson defined "essentially positive" tensors as Definition 3. By the general product of tensors, Shao obtained Proposition 4 and Definition 5 which is equivalent to Definition 3.

Definition 3 (see [6], Definition 3.1). A nonnegative tensor $\mathbb{A}$ is called essentially positive, if, for any nonnegative nonzero vector $x \in \mathbb{R}^{n}, \mathbb{A} \cdot x>0$ holds.

Proposition 4 (see [5], Proposition 4.1). Let A be an order $m$ and dimension $n$ nonnegative tensor. Then the following three conditions are equivalent:

(1) For any $i, j \in[n], a_{i j \ldots j}>0$ holds.

(2) For any $j \in[n], \mathbb{A} \cdot e_{j}>0$ holds (where $e_{j}$ is the $j$-th column of the identity matrix $I_{n}$ ). holds.

(3) For any nonnegative nonzero vector $x \in \mathbb{R}^{n}, \mathbb{A} \cdot x>0$

Definition 5 (see [5], Definition 4.1). A nonnegative tensor $\mathbb{A}$ is called essentially positive, if it satisfies one of the three conditions in Proposition 4.

Based on the above arguments and the zero patterns defined by Shao in [5], Shao showed a characterization of primitive tensors and defined the primitive degree as follows.

Proposition 6 (see [5], Theorem 4.1). A nonnegative tensor $\mathbb{A}$ is primitive if and only if there exists some positive integer $r$ such that $\mathbb{A}^{r}$ is essentially positive. Furthermore, the smallest such $r$ is the primitive degree of $\mathbb{A}, \gamma(\mathbb{A})$.

The concept of the majorization matrix of a tensor introduced by Pearson is very useful.

Definition 7 (see [6], Definition 2.1). The majorization matrix $M(\mathbb{A})$ of the tensor $\mathbb{A}$ is defined as $(M(\mathbb{A}))_{i j}=a_{i j \ldots j}$ for $i, j \in$ $[n]$.
By Definition 5, Proposition 6, and Definition 7, the following characterization of the primitive tensors was easily obtained.

Proposition 8 (see [7], Remark 2.6). Let $\mathbb{A}$ be a nonnegative tensor with orderm and dimensionn. Then $\mathbb{A}$ is primitive if and only if there exists some positive integer $r$ such that $M\left(\mathbb{A}^{r}\right)>0$. Furthermore, the smallest such $r$ is the primitive degree of $\mathbb{A}$, $\gamma(\mathbb{A})$.

On the primitive degree $\gamma(\mathbb{A})$, Shao proposed the following conjecture for further research.

Conjecture 9 (see [5], Conjecture 1). When $m$ is fixed, then there exists some polynomial $f(n)$ on $n$ such that $\gamma(\mathbb{A}) \leq f(n)$ for all nonnegative primitive tensors of order $m$ and dimension $n$.

In the case of $m=2$ ( $\mathbb{A}$ is a matrix), the well-known Wielandt upper bound tells us that we can take $f(n)=(n-$ $1)^{2}+1$. Recently, the authors [7] confirmed Conjecture 9 by proving Theorem 10.

Theorem 10 (see [7], Theorem 1.2). Let A be a nonnegative primitive tensor with order $m$ and dimension $n$. Then its primitive degree $\gamma(\mathbb{A}) \leq(n-1)^{2}+1$, and the upper bound is tight.

They also showed that there are no gaps in the tensor case in [8], which implies that the result of the case $m \geq 3$ is totally different from the case $m=2$ ( $\mathbb{A}$ is a matrix). In [5], Shao also proposed the concept of strongly primitive tensor for further research.

Definition 11 (see [5], Definition 4.3). Let $\mathbb{A}$ be a nonnegative tensor with order $m$ and dimension $n$. If there exists some positive integer $k$ such that $\mathbb{A}^{k}>0$ is a positive tensor, then $\mathbb{A}$ is called strongly primitive, and the smallest such $k$ is called the strongly primitive degree of $A$.

Let $\mathbb{A}=\left(a_{i_{1} i_{2} \ldots i_{m}}\right)$ be a nonnegative tensor with order $m$ and dimension $n$. It is clear that if $\mathbb{A}$ is strongly primitive, then $\mathbb{A}$ is primitive. For convenience, let $\eta(\mathbb{A})$ be the strongly primitive degree of $\mathbb{A}$. Clearly, $\gamma(\mathbb{A}) \leq \eta(\mathbb{A})$. In fact, it is obvious that, in the matrix case $(m=2)$, a nonnegative matrix $A$ is primitive if and only if $A$ is strongly primitive, and $\gamma(A)=\eta(A)=\exp (A)$. But in the case $m \geq 3$ Shao gave an example to show that these two concepts are not equivalent. In [8], the authors proposed the following question.

Question 12 ([8], Question 4.18). Can we define and study the strongly primitive degree, the strongly primitive degree set, the $j$-strongly primitive degree of strongly primitive tensors and so on?

Based on Question 12, we study primitive tensors and strongly primitive tensors in this paper, show that an order $m$ dimension 2 tensor is primitive if and only if its majorization matrix is primitive, and obtain the characterization of order $m$ dimension 2 strongly primitive tensors and the bound of the strongly primitive degree. Furthermore, we study the 
properties of strongly primitive tensors with $n \geq 3$ and propose some problems for further research.

\section{Preliminaries}

In [8], the authors obtained the following Proposition 13 and gave Example 15 by computing the strongly primitive degree.

Proposition 13 ([8], Proposition 4.16). Let $\mathbb{A}=\left(a_{i_{1} i_{2} \ldots i_{m}}\right)$ be a nonnegative strongly primitive tensor with order $m$ and dimension $n$. Then for any $\alpha \in[n]^{m-1}$, there exists some $i \in[n]$ such that $a_{i \alpha}>0$.

Let $k(\geq 0), n(\geq 2), q(\geq 0), r(\geq 1)$ be integers and $k=$ $(n-1) q+r$ with $1 \leq r \leq n-1$ when $k \geq 1$. In [7-9], the authors defined some nonnegative tensors with order $m$ and dimension $n$ as follows:

$$
\mathbb{A}_{k}=\left(a_{i_{1} i_{2} \cdots i_{m}}^{[k]}\right)_{1 \leq i_{j} \leq n(j=1, \ldots, m)},
$$

where one has the following:

(1)

$$
M\left(\mathbb{A}_{k}\right)=M_{1}=\left(\begin{array}{cccccc}
0 & 0 & \cdots & 0 & 1 & 1 \\
1 & 0 & \cdots & 0 & 0 & 0 \\
0 & 1 & \cdots & 0 & 0 & 0 \\
\vdots & \vdots & \ddots & \vdots & \vdots & \vdots \\
0 & 0 & \cdots & 1 & 0 & 0 \\
0 & 0 & \cdots & 0 & 1 & 0
\end{array}\right) .
$$

(2) $a_{i i_{2} \ldots i_{m}}^{[0]}=0$, if $i_{2} \ldots i_{m} \neq i_{2} \ldots i_{2}$ for any $i \in[n]$.

(3) $a_{i \alpha}^{[k]}=1$, if $i \in[n] \backslash\{r-q, r-q+1, \ldots, r, r+1\}(\bmod n)$ and $\alpha=i_{2} \ldots i_{m} \in[n]^{m-1}$ with $\left\{i_{2}, \ldots, i_{m}\right\}=\{r-q-$ $1, r\}(\bmod n)$.

(4) $a_{i_{1} i_{2} \ldots i_{m}}^{[k]}=0$, except for (1) and (3).

The authors showed the tensors $\mathbb{A}_{k}(k \geq 0)$ are primitive, the primitive degree $\gamma\left(\mathbb{A}_{0}\right)=(n-1)^{2}+1([7])$ and $\gamma\left(\mathbb{A}_{k}\right)=k+n$ ([8], Theorem 3.3) for $1 \leq k \leq n^{2}-3 n+2$.

Remark 14. It is clear that, for any $\mathbb{A}_{k}\left(0 \leq k \leq n^{2}-3 n+2\right)$, there exists some $\alpha \in[n]^{m-1}$, for any $i \in[n], a_{i \alpha}^{[k]}=0$. Thus, for each $0 \leq k \leq n^{2}-3 n+2, \mathbb{A}_{k}$ is not a strongly primitive tensor by Proposition 13.

Example 15 ([8], Example 4.17). Let $m=n=3$, and let $\mathbb{A}=$ $\left(a_{i_{1} i_{2} i_{3}}\right)$ be a nonnegative tensor with order $m$ and dimension $n$, where $a_{111}=a_{222}=a_{333}=a_{233}=a_{311}=0$ and other $a_{i_{1} i_{2} i_{3}}=1$. Then $\eta(\mathbb{A})=4$.

Remark 16. In fact, we can obtain $\gamma(\mathbb{A})=\eta(\mathbb{A})=4$ because of $a_{233333333}^{(3)}=0$, where $\mathbb{A}^{3}=\left(a_{i_{1} i_{2} \ldots i_{9}}^{(3)}\right)$.

In the computation of Example 15, we note that the following equation is useful and will be used repeatedly. It is not difficult to obtain the equation by the general product of two $\mathrm{n}$-dimensional tensors which is defined in Definition 1.2 in [5].

Let $\mathbb{A}$ be a nonnegative primitive tensor with order $m$ and dimension $n, \alpha_{2}, \ldots, \alpha_{m} \in[n]^{(m-1)^{k-1}}$. Then we have

$$
\begin{aligned}
& \left(\mathrm{A}^{k}\right)_{i \alpha_{2} \cdots \alpha_{m}} \\
& =\sum_{i_{2}, i_{3}, \ldots, i_{m}=1}^{n} a_{i i_{2} i_{3} \cdots i_{m}}\left(\mathrm{~A}^{k-1}\right)_{i_{2} \alpha_{2}} \cdots\left(\mathrm{A}^{k-1}\right)_{i_{m} \alpha_{m}} .
\end{aligned}
$$

Proposition 17 (see [7], Proposition 2.7). Let $\mathbb{A}$ be a nonnegative primitive tensor with order $m$ and dimension $n$ and $M(\mathbb{A})$ be the majorization matrix of $A$. Then we have the following:

(1) For each $j \in[n]$, there exists an integer $i \in[n] \backslash\{j\}$ such that $(M(\mathbb{A}))_{i j}>0$.

(2) There exist some $j \in[n]$ and integers $u, v$ with $1 \leq u<$ $v \leq n$ such that $(M(\mathbb{A}))_{u j}>0$ and $(M(\mathbb{A}))_{v j}>0$.

Let $\alpha=j j \ldots j \in[n]^{m-1}$; then $M(\mathbb{A})_{i j}=a_{i \alpha}$. We can see that Proposition 13 is the generalization of result (1) of Proposition 17 from a primitive tensor to a strongly primitive tensor. We note that Proposition 17 played an important role in [7], and if $A$ is a nonnegative strongly primitive tensor, then A must be a nonnegative primitive tensor; thus result (2) of Proposition 17 also holds for nonnegative strongly primitive tensors.

Proposition 18. Let $\mathbb{A}=\left(a_{i_{1} i_{2} \ldots i_{m}}\right)$ be a nonnegative strongly primitive tensor with order $m$ and dimension $n$. Then there exists at least one $j \in[n]$ and integers $u, v$ with $1 \leq u<v \leq n$ such that $(M(\mathbb{A}))_{u j}>0$ and $(M(\mathbb{A}))_{v j}>0$.

Proposition 19. Let $\mathbb{A}=\left(a_{i_{1} i_{2} \ldots i_{m}}\right)_{1 \leq i_{j} \leq n(j=1, \ldots, m)}$ be a nonnegative tensor with order $m$ and dimension $n$ and $\mathbb{A} \neq \sqrt{ }$. For given $i \in[n]$, if $a_{i \alpha}=a_{j i i \ldots i}=1$ for any $\alpha \in[n]^{m-1}$ and any $j \in[n] \backslash\{i\}$, then $\mathbb{A}$ is strongly primitive with $\eta(\mathbb{A})=2$.

Proof. By (7), for any $k \in[n]$ and $\alpha_{2}, \ldots, \alpha_{m} \in[n]^{m-1}$, we have

$\left(\mathbb{A}^{2}\right)_{k \alpha_{2} \ldots \alpha_{m}}=\sum_{k_{2}, k_{3}, \ldots, k_{m}=1}^{n} a_{k k_{2} \ldots k_{m}} a_{k_{2} \alpha_{2}} \ldots a_{k_{m} \alpha_{m}} \geq$ $a_{k i \ldots . . .} a_{i \alpha_{2}} \ldots a_{i \alpha_{m}}=1$, which implies $\mathbb{A}$ is strongly primitive and $\eta(\mathrm{A})=2$.

Remark 20. From Proposition 19, we can see the following:

(1) There exist at least $n\left(2^{(n-1)\left(n^{m-1}-1\right)}-1\right)$ strongly primitive tensors such that their strongly primitive degree is equal to 2 .

(2) We cannot improve the result of Proposition 13 any more by the fact that there exists $i \in[n]$ such that $a_{i \alpha}=1>0$ for any $\alpha \in[n]^{m-1}$ and there is exactly one $i$ such that $a_{i \alpha}>0$ for any $\alpha \neq i i \ldots i$.

(3) Similarly, we cannot improve the result of Proposition 18 any more by the fact that there is exactly one $i \in[n]$ such that $(M(\mathbb{A}))_{u i}>0$ for any $u \in[n]$ and for any other $j \in[n] \backslash\{i\}$, there exists only $i \in[n]$ such that $(M(A))_{i j}>0$.

(4) What is more, combining the above arguments, we know whether a nonnegative tensor is a nonnegative strongly 
primitive tensor or not, and the values of the strongly primitive degree of a nonnegative strongly primitive tensor do not depend on the number of nonzero entries but the positions of the nonzero entries.

Proposition 21. Let $\mathbb{A}=\left(a_{i_{1} i_{2} \ldots i_{m}}\right)$ be a nonnegative strongly primitive tensor with order $m$ and dimension $n$. Then for any $i \in[n]$, there exists some $\alpha \in[n]^{m-1}$ such that $a_{i \alpha}>0$.

Proof. Since $\mathbb{A}$ is strongly primitive, there exists some $k>0$ such that $\mathbb{A}^{k}>0$ by Definition 1 . Assume that there exists some $i \in[n]$ such that $a_{i \alpha}=0$ for any $\alpha \in[n]^{m-1}$. Then by (7), we have

$$
\begin{aligned}
& \left(\mathrm{A}^{k}\right)_{i \alpha_{2} \cdots \alpha_{m}} \\
& \quad=\sum_{i_{2}, i_{3}, \ldots, i_{m}=1}^{n} a_{i i_{2} i_{3} \cdots i_{m}}\left(\mathbb{A}^{k-1}\right)_{i_{2} \alpha_{2}} \cdots\left(\mathbb{A}^{k-1}\right)_{i_{m} \alpha_{m}}=0
\end{aligned}
$$

which leads to a contraction.

Remark 22. Let $\mathbb{A}=\left(a_{i_{1} i_{2} \ldots i_{m}}\right)_{1 \leq i_{j} \leq n(j=1, \ldots, m)}$ be a nonnegative tensor with order $m$ and dimension $n$. For given $i \in[n]$, we take $a_{i \alpha}=a_{j i i \ldots i}=1$ for any $\alpha \in[n]^{m-1}$ and any $j \in[n] \backslash\{i\}$ and any other entry $a_{i_{1} i_{2} \ldots i_{m}}=0$. Then $\mathbb{A}$ is strongly primitive with $\eta(\mathbb{A})=2$ by Proposition 19. This implies that we cannot improve the result of Proposition 21 anymore, and it indicates the importance of the positions of the nonzero entries again.

Proposition 23. Let $\mathbb{A}$ be a nonnegative strongly primitive tensor and $k=\eta(\mathbb{A})$. Then, for any integer $t>k>0$, we have $\mathbb{A}^{t}>0$.

Proof. It is clear that $\mathbb{A}^{k}>0$ by $k=\eta(\mathbb{A})$. We only need to show $\mathbb{A}^{k+1}>0$; say, for any $i \in[n]$ and any $\alpha_{2}, \ldots, \alpha_{m} \in$ $[n]^{(m-1)^{k}}$, we show $\left(\mathbb{A}^{k+1}\right)_{i \alpha_{2} \ldots \alpha_{m}}>0$.

By Proposition 21, there exists some $\alpha=j_{2} j_{3} \ldots j_{m} \in$ $[n]^{m-1}$ such that $a_{i \alpha}=a_{i j_{2} \ldots j_{m}}>0$. By $A^{k}>0$ we have $\left(\mathbb{A}^{k}\right)_{j_{2} \alpha_{2}}>0, \ldots,\left(\mathbb{A}^{k}\right)_{j_{m} \alpha_{m}}>0$; then by (7), we have

$$
\begin{aligned}
& \left(\mathbb{A}^{k+1}\right)_{i \alpha_{2} \cdots \alpha_{m}} \\
& =\sum_{i_{2}, i_{3}, \cdots, i_{m}=1}^{n} a_{i i_{2} i_{3} \cdots i_{m}}\left(\mathbb{A}^{k}\right)_{i_{2} \alpha_{2}}\left(\mathbb{A}^{k}\right)_{i_{3} \alpha_{3}} \cdots\left(\mathbb{A}^{k}\right)_{i_{m} \alpha_{m}} \\
& \geq a_{i j_{2} j_{3} \cdots j_{m}}\left(\mathbb{A}^{k}\right)_{j_{2} \alpha_{2}} \ldots\left(\mathbb{A}^{k}\right)_{j_{m} \alpha_{m}}>0 .
\end{aligned}
$$

Proposition 24. Let $\mathbb{A}$ be a nonnegative tensor with order $m$ and dimension $n$ and $t$ be a positive integer. Then $\mathbb{A}$ is strongly primitive if and only if $\mathbb{A}^{t}$ is strongly primitive.

Proof. Firstly, the sufficiency is obvious. Now we show the necessity. Let $k=\eta(\mathbb{A})$. Then $\mathbb{A}^{k}>0$ by $\mathbb{A}$ is strongly primitive. Let $s$ be a positive integer such that $s t \geq k$; then $\mathbb{A}^{s t}>0$ by Proposition 23. Thus $\left(\mathbb{A}^{t}\right)^{s}=\mathbb{A}^{s t}>0$, which implies $\mathbb{A}^{t}$ is strongly primitive.

\section{A Characterization of the (Strongly) Primitive Tensor with Order $m$ and Dimension 2}

In this section, we study primitive tensors and strongly primitive tensors in this paper, show that an order $m$ dimension 2 tensor is primitive if and only if its majorization matrix is primitive, and obtain the characterization of order $m$ dimension 2 strongly primitive tensors and the bound of the strongly primitive degree.

Lemma 25 (see [5], Corollary 4.1). Let $\mathbb{A}$ be a nonnegative tensor with order $m$ and dimension $n$. If $M(\mathbb{A})$ is primitive, then $\mathbb{A}$ is also primitive and in this case we have $\gamma(\mathbb{A}) \leq$ $\gamma(M(\mathbb{A})) \leq(n-1)^{2}+1$.

Theorem 26. Let $\mathbb{A}$ be a nonnegative tensor with order $m$ and dimension $n=2$. Then $\mathbb{A}$ is primitive if and only if $M(\mathbb{A})$ is primitive.

Proof. Firstly, the sufficiency is obvious by Lemma 25. Now we only show the necessity. Clearly, all primitive $(0,1)$ matrices of order 2 are listed as follows:

$$
\begin{aligned}
& \left(\begin{array}{ll}
1 & 1 \\
1 & 0
\end{array}\right), \\
& \left(\begin{array}{ll}
0 & 1 \\
1 & 1
\end{array}\right), \\
& \left(\begin{array}{ll}
1 & 1 \\
1 & 1
\end{array}\right) .
\end{aligned}
$$

Let $\mathbb{A}$ be primitive. Then $\gamma(\mathbb{A}) \leq 2$ by Theorem 10 and $M\left(\mathbb{A}^{2}\right)>0$ by Proposition 8 . Now we assume that $M(\mathbb{A})$ is not primitive; we will show $\mathbb{A}$ is also not primitive.

It is not difficult to find that

$$
\begin{aligned}
& \left(\mathbb{A}^{2}\right)_{i j j \cdots j}=\sum_{i_{2}, i_{3}, \ldots, i_{m}=1}^{2} a_{i i_{2} i_{3} \cdots i_{m}} a_{i_{2} j j \cdots j} \cdots a_{i_{m} j j \cdots j} \\
& =a_{i 22 \cdots 2}\left(a_{2 j j \cdots j}\right)^{m-1} \\
& \quad+\sum_{i_{2}, i_{3}, \ldots, i_{m}=1, i_{2} i_{3} \cdots i_{m} \neq 22 \cdots 2}^{2} a_{i i_{2} \cdots i_{m}} a_{i_{2} j j \cdots j} \cdots a_{i_{m} j j \cdots j} \\
& =a_{i 11 \cdots 1}\left(a_{1 j j \cdots j}\right)^{m-1} \\
& \quad+\sum_{i_{2}, i_{3}, \ldots, i_{m}=1, i_{2} i_{3} \cdots i_{m} \neq 11 \cdots 1}^{2} a_{i i_{2} \cdots i_{m}} a_{i_{2} j j \cdots j} \cdots a_{i_{m} j j \cdots j} .
\end{aligned}
$$

In (12), we note that $i_{2} i_{3} \ldots i_{m} \neq 22 \ldots 2$, which implies that there exists at least one entry, say, $i_{s}=1$, where $2 \leq s \leq m$; then $a_{1 j j \ldots j} \in\left\{a_{i_{2} j j \ldots j}, \ldots, a_{i_{m} j j \ldots j}\right\}$.

Similarly, in (13), we note that $i_{2} i_{3} \ldots i_{m} \neq 11 \ldots 1$, which implies that there exists at least one entry, say, $i_{s}=2$, where $2 \leq s \leq m$; then $a_{2 j j \ldots j} \in\left\{a_{i_{2} j j \ldots j}, \ldots, a_{i_{m} j j \ldots j}\right\}$. 
Thus, by (12), (13), and the above arguments, we have

$$
\begin{aligned}
M\left(\mathbb{A}^{2}\right)_{i j} & =\left(\mathbb{A}^{2}\right)_{i j j \cdots j}=a_{i 22 \cdots 2}\left(a_{2 j j \cdots j}\right)^{m-1}+a_{1 j j \cdots j} P \\
& =a_{i 11 \cdots 1}\left(a_{1 j j \cdots j}\right)^{m-1}+a_{2 j j \cdots j} Q .
\end{aligned}
$$

Since $M(\mathbb{A})$ is not primitive; by $(10)$, we can complete the proof by the following two cases.

Case 1. $M(\mathbb{A})=\left(\begin{array}{ll}* & 0 \\ * & *\end{array}\right)$ or $\left(\begin{array}{ll}* & * \\ 0 & *\end{array}\right)$.

Subcase 1.1.M(A) $)_{12}=0$.

Then $a_{12 \ldots 2}=0$. By $(14)$, we have $M\left(\mathbb{A}^{2}\right)_{12}=\left(\mathbb{A}^{2}\right)_{12 \ldots 2}=0$, which implies $\mathbb{A}^{2}$ is not essentially positive.

Subcase 1.2.M(A) $)_{21}=0$.

Then $a_{21 \ldots 1}=0$. By $(15)$, we have $M\left(\mathbb{A}^{2}\right)_{21}=\left(\mathbb{A}^{2}\right)_{21 \ldots 1}=0$, which implies $\mathbb{A}^{2}$ is not essentially positive.

Case 2. $M(\mathbb{A})=\left(\begin{array}{ll}0 & 1 \\ 1 & 0\end{array}\right)$.

Then we have $M(\mathbb{A})_{11}=M(\mathbb{A})_{22}=0$; that is, $a_{11 \ldots 1}=$ $a_{22 \ldots 2}=0$; by $(15)$ we have $M\left(\mathbb{A}^{2}\right)_{12}=\left(\mathbb{A}^{2}\right)_{12 \ldots 2}=0$, which implies $\mathbb{A}^{2}$ is not essentially positive.

Based on the above two cases and Proposition 6, we complete the proof of the necessity.

A nature question is whether the result of Theorem 26 is true for $n \geq 3$ or not. The following Example 27 shows that the necessity of Theorem 26 is false with $n \geq 3$.

Example 27. Let $\mathbb{A}=\left(a_{i_{1} i_{2} \ldots i_{m}}\right)$ be a nonnegative tensor of order $m$ and dimension $n \geq 3$, where

$$
a_{i_{1} i_{2} \cdots i_{m}}= \begin{cases}0, & \text { if } i_{1}=1, i_{2}=i_{3}=\cdots=i_{m} \neq 1 \\ 1, & \text { otherwise. }\end{cases}
$$

Then $\mathbb{A}$ is (strongly) primitive, but $M(\mathbb{A})$ is not primitive.

Proof. By direct calculation and Definition 2, we know that $\mathbb{A}^{2}$ is the tensor of order $(m-1)^{2}+1$ and dimension $n$, and for any $1 \leq i \leq n$, we have

$$
\begin{gathered}
\left(\mathbb{A}^{2}\right)_{i \alpha_{2} \alpha_{3} \cdots \alpha_{m}}=\sum_{i_{2}, i_{3}, \ldots, i_{m}=1}^{2} a_{i i_{2} i_{3} \cdots i_{m}} a_{i_{2} \alpha_{2}} \cdots a_{i_{m} \alpha_{m}} \\
\geq \begin{cases}a_{12 \cdots m} a_{2 \alpha_{2}} \cdots a_{m \alpha_{m}}=1, & \text { if } i=1 ; \\
a_{i i \cdots i} a_{i \alpha_{2}} \cdots a_{i \alpha_{m}}=1, & \text { otherwise. }\end{cases}
\end{gathered}
$$

Obviously, $\mathbb{A}^{2}$ is positive; then $\mathbb{A}$ is strongly primitive with $\eta(\mathbb{A})=2$ and thus $\mathbb{A}$ is primitive with $\gamma(\mathbb{A})=2$.

On the other hand, by the definition of $\mathbb{A}$, we have

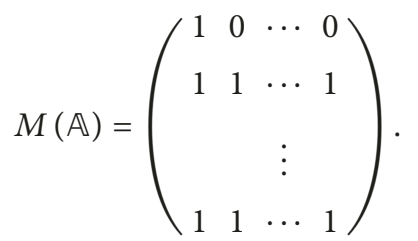

Since the associated digraph of $M(\mathbb{A})$ is not strongly connected, thus $M(\mathbb{A})$ is not primitive.

Next, we will study the strongly primitive degree of order $m$ and dimension 2 tensors. Firstly, we discuss an example with order $m=5$ and dimension $n=2$ tensor as follows.

Definition 28 (see [10]). Let $\mathbb{A}$ be a tensor with order $m$ and dimension $n$. The $i$-th slice of $\mathbb{A}$, denoted by $\mathbb{A}[i]$, is the subtensor of $\mathbb{A}$ with order $m-1$ and dimension $n$ such that $(\mathbb{A}[i])_{i_{2} \ldots i_{m}}=a_{i i_{2} \ldots i_{m}}$.

Example 29. Let $\mathbb{A}=\left(a_{i_{1} i_{2} i_{3} i_{4} i_{5}}\right)_{1 \leq i_{j} \leq 2(j=1, \ldots, 5)}$ be a nonnegative tensor with order $m=5$ and dimension $n=2$, where $a_{12122}=a_{21121}=0$ and other $a_{i_{1} i_{2} i_{3} i_{4} i_{5}}=1$. Then there exists at least one zero element in each slice of $A^{2}$.

Proof. Let $\alpha_{1}=2122, \alpha_{2}=1121$, and denote $\beta_{2}=\beta_{4}=\beta_{5}=$ $\alpha_{1}, \beta_{3}=\alpha_{2}$. Then we have

$$
\begin{aligned}
& \left(A^{2}\right)_{1 \beta_{2} \beta_{3} \beta_{4} \beta_{5}} \\
& =\sum_{i_{2}, i_{3}, i_{4}, i_{5}=1}^{2} a_{1 i_{2} i_{3} i_{4} i_{5}} a_{i_{2} \beta_{2}} a_{i_{3} \beta_{3}} a_{i_{4} \beta_{4}} a_{i_{5} \beta_{5}} \\
& =\sum_{i_{3}, i_{4}, i_{5}=1}^{2} a_{11 i_{3} i_{4} i_{5}} a_{1 \beta_{2}} a_{i_{3} \beta_{3}} a_{i_{4} \beta_{4}} a_{i_{5} \beta_{5}} \\
& +\sum_{i_{3}, i_{4}, i_{5}=1}^{2} a_{12 i_{3} i_{4} i_{5}} a_{2 \beta_{2}} a_{i_{3} \beta_{3}} a_{i_{4} \beta_{4}} a_{i_{5} \beta_{5}} \\
& \stackrel{a_{1 \beta_{2}}=0}{=} \sum_{i_{3}, i_{4}, i_{5}=1}^{2} a_{12 i_{3} i_{4} i_{5}} a_{2 \beta_{2}} a_{i_{3} \beta_{3}} a_{i_{4} \beta_{4}} a_{i_{5} \beta_{5}} \\
& =\sum_{i_{4}, i_{5}=1}^{2} a_{121 i_{4} i_{5}} a_{2 \beta_{2}} a_{1 \beta_{3}} a_{i_{4} \beta_{4}} a_{i_{5} \beta_{5}} \\
& +\sum_{i_{4}, i_{5}=1}^{2} a_{122 i_{4} i_{5}} a_{2 \beta_{2}} a_{2 \beta_{3}} a_{i_{4} \beta_{4}} a_{i_{5} \beta_{5}} \\
& \underline{\underline{a_{2 \beta_{3}}=0}} \sum_{i_{4}, i_{5}=1}^{2} a_{121 i_{4} i_{5}} a_{2 \beta_{2}} a_{1 \beta_{3}} a_{i_{4} \beta_{4}} a_{i_{5} \beta_{5}} \\
& =\sum_{i_{5}=1}^{2} a_{1211 i_{5}} a_{2 \beta_{2}} a_{1 \beta_{3}} a_{1 \beta_{4}} a_{i_{5} \beta_{5}} \\
& +\sum_{i_{5}=1}^{2} a_{1212 i_{5}} a_{2 \beta_{2}} a_{1 \beta_{3}} a_{2 \beta_{4}} a_{i_{5} \beta_{5}} \\
& \stackrel{a_{1 \beta_{4}}=0}{=} \sum_{i_{5}=1}^{2} a_{1212 i_{5}} a_{2 \beta_{2}} a_{1 \beta_{3}} a_{2 \beta_{4}} a_{i_{5} \beta_{5}} \\
& =a_{12121} a_{2 \beta_{2}} a_{1 \beta_{3}} a_{2 \beta_{4}} a_{1 \beta_{5}}
\end{aligned}
$$




$$
\begin{gathered}
+a_{12122} a_{2 \beta_{2}} a_{1 \beta_{3}} a_{2 \beta_{4}} a_{2 \beta_{5}} \\
\stackrel{a_{1 \beta_{5}}=0}{=} a_{12122} a_{2 \beta_{2}} a_{1 \beta_{3}} a_{2 \beta_{4}} a_{2 \beta_{5}} \stackrel{a_{12122}=0}{=} 0 .
\end{gathered}
$$

Similarly, we let $\gamma_{2}=\gamma_{3}=\gamma_{5}=\alpha_{2}$ and $\gamma_{4}=\alpha_{1}$, we can show $\left(\mathbb{A}^{2}\right)_{2 \gamma_{2}} \gamma_{3} \gamma_{4} \gamma_{5}=0$, and we omit it.

Combining the above arguments, we know there exists at least one zero element in each slice of $\mathbb{A}^{2}$ by $\left(A^{2}\right)_{1 \beta_{2} \beta_{3} \beta_{4} \beta_{5}}=$ $\left(\mathbb{A}^{2}\right)_{2 \gamma_{2} \gamma_{3} \gamma_{4} \gamma_{5}}=0$.

Similarly, the result of Example 29 can be generalized to any nonnegative tensor with order $m$ and dimension $n=2$.

Lemma 30. Let $\mathbb{A}=\left(a_{i_{1} i_{2} \ldots i_{m}}\right)_{1 \leq i_{j} \leq 2(j=1, \ldots, m)}$ be a nonnegative tensor with order $m$ and dimension $n=2$. If there exist $\alpha_{1}=$ $j_{2} j_{3} \ldots j_{m} \in[2]^{m-1}$ and $\alpha_{2}=k_{2} k_{3} \ldots k_{m} \in[2]^{m-1}$ such that

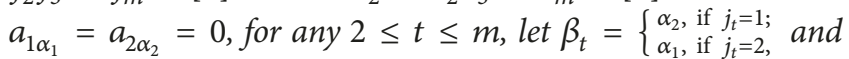

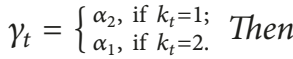

$$
\begin{aligned}
& \left(\mathbb{A}^{2}\right)_{1 \beta_{2} \cdots \beta_{m}}=0, \\
& \left(\mathbb{A}^{2}\right)_{2 \gamma_{2} \cdots \gamma_{m}}=0 .
\end{aligned}
$$

Proof. We first show $\left(\mathbb{A}^{2}\right)_{1 \beta_{2} \ldots \beta_{m}}=0$. For any $2 \leq t \leq m$, $j_{t} \in\{1,2\}$, we denote $\overline{j_{t}} \in\{1,2\} \backslash\left\{j_{t}\right\}$. Then we have $a_{\bar{j}_{t} \beta_{t}}=0$ by $a_{1 \alpha_{1}}=a_{2 \alpha_{2}}=0$, and

$$
\begin{aligned}
& \left(\mathbb{A}^{2}\right)_{1 \beta_{2} \cdots \beta_{m}}=\sum_{i_{2}, i_{3}, \ldots, i_{m}=1}^{2} a_{1 i_{2} i_{3} \cdots i_{m}} a_{i_{2} \beta_{2}} \cdots a_{i_{m} \beta_{m}} \\
& =\sum_{i_{3}, \ldots, i_{m}=1}^{2} a_{11 i_{3} \cdots i_{m}} a_{1 \beta_{2}} a_{i_{3} \beta_{3}} \cdots a_{i_{m} \beta_{m}} \\
& +\sum_{i_{3}, \ldots, i_{m}=1}^{2} a_{12 i_{3} \cdots i_{m}} a_{2 \beta_{2}} a_{i_{3} \beta_{3}} \cdots a_{i_{m} \beta_{m}} \\
& \stackrel{a_{\overline{j_{2} \beta_{2}}}=0}{=} \sum_{i_{3}, \ldots, i_{m}=1}^{2} a_{1 j_{2} i_{3} \cdots i_{m}} a_{j_{2} \beta_{j_{2}}} a_{i_{3} \beta_{3}} \cdots a_{i_{m} \beta_{m}} \\
& =\sum_{i_{4}, \ldots, i_{m}=1}^{2} a_{1 j_{2} 1 i_{4} \cdots i_{m}} a_{j_{2} \beta_{j_{2}}} a_{1 \beta_{3}} a_{i_{4} \beta_{4}} \cdots a_{i_{m} \beta_{m}} \\
& +\sum_{i_{4}, \ldots, i_{m}=1}^{2} a_{1 j_{2} 2 i_{4} \cdots i_{m}} a_{j_{2} \beta_{j_{2}}} a_{2 \beta_{3}} a_{i_{4} \beta_{4}} \cdots a_{i_{m} \beta_{m}} \\
& \stackrel{a_{\overline{j_{3} \beta_{3}}}=0}{=} \sum_{i_{4}, \ldots, i_{m}=1}^{2} a_{1 j_{2} j_{3} i_{4} \cdots i_{m}} a_{j_{2} \beta_{j_{2}}} a_{j_{3} \beta_{j_{3}}} a_{i_{4} \beta_{4}} \cdots a_{i_{m} \beta_{m}} \\
& =\sum_{i_{m}=1}^{2} a_{1 j_{2} j_{3} \cdots j_{m-1} i_{m}} a_{j_{2} \beta_{j_{2}}} a_{j_{3} \beta_{j_{3}}} \cdots a_{j_{m-1} \beta_{j_{m-1}}} a_{i_{m} \beta_{m}}
\end{aligned}
$$

$$
\begin{aligned}
& =a_{1 j_{2} \cdots j_{m-1} 1} a_{j_{2} \beta_{j_{2}}} \cdots a_{j_{m-1} \beta_{j_{m-1}}} a_{1 \beta_{m}} \\
& +a_{1 j_{2} \cdots j_{m-1} 2} a_{j_{2} \beta_{j_{2}}} \cdots a_{j_{m-1} \beta_{j_{m-1}}} a_{2 \beta_{m}} \\
& \stackrel{a_{\overline{j_{m}} \beta_{m}}=0}{=} a_{1 j_{2} \cdots j_{m}} a_{j_{2} \beta_{j_{2}}} \cdots a_{j_{m} \beta_{j_{m}}} \stackrel{a_{1 \alpha_{1}}=0}{=} 0 .
\end{aligned}
$$

Similarly, for any $2 \leq t \leq m, k_{t} \in\{1,2\}$, we denote $\overline{k_{t}} \in\{1,2\} \backslash\left\{k_{t}\right\}$. Then we have $a_{\overline{k_{t}} \gamma_{t}}=0$ and we can show $\left(\mathbb{A}^{2}\right)_{2 \gamma_{2} \ldots \gamma_{m}}=0$ by

$$
\left(\mathbb{A}^{2}\right)_{2 \gamma_{2} \cdots \gamma_{m}}=\sum_{i_{2}, i_{3}, \ldots, i_{m}=1}^{2} a_{2 i_{2} i_{3} \cdots i_{m}} a_{i_{2} \gamma_{2}} \cdots a_{i_{m} \gamma_{m}}
$$

and similar process of the above arguments. Thus we complete the proof of (20).

Theorem 31. Let $\mathbb{A}=\left(a_{i_{1} i_{2} \ldots i_{m}}\right)_{1 \leq i_{j} \leq 2(j=1, \ldots, m)}$ be a nonnegative tensor with order $m$ and dimension $n=2$. If there exist $\alpha_{1}=$ $j_{2} j_{3} \ldots j_{m} \in[2]^{m-1}$ and $\alpha_{2}=k_{2} k_{3} \ldots k_{m} \in[2]^{m-1}$ such that $a_{1 \alpha_{1}}=a_{2 \alpha_{2}}=0$, then $\mathbb{A}$ is not strongly primitive.

Proof. Now we show that there exists at least one zero element in each slice of $\mathbb{A}^{r}$ by induction on $r(\geq 2)$.

Firstly, by Lemma 30, we know there exists at least one zero element in each slice of $\mathbb{A}^{2}$. Now we assume that there exists at least one zero element in each slice of $A^{r-1}$; say, there exist $\delta_{1}, \delta_{2} \in[2]^{(m-1)^{r-1}}$ such that $\left(\mathbb{A}^{r-1}\right)_{1 \delta_{1}}=\left(\mathbb{A}^{r-1}\right)_{2 \delta_{2}}=0$. For any $2 \leq t \leq m$, let $\beta_{t}=\left\{\begin{array}{l}\delta_{2} \text { if } j_{t}=1 \\ \delta_{1} \text { if } j_{t}=2\end{array}\right.$ and $\gamma_{t}= \begin{cases}\delta_{2} & \text { if } k_{t}=1 \\ \delta_{1} \text { if } k_{t}=2\end{cases}$ Then by (7) and the similar proof of Lemma 30, we have

$$
\begin{aligned}
& \left(\mathbb{A}^{r}\right)_{1 \beta_{2} \cdots \beta_{m}} \\
& \quad=\sum_{i_{2}, i_{3}, \ldots, i_{m}=1}^{n} a_{1 i_{2} i_{3} \cdots i_{m}}\left(\mathbb{A}^{r-1}\right)_{i_{2} \beta_{2}} \cdots\left(\mathbb{A}^{r-1}\right)_{i_{m} \beta_{m}}=0,
\end{aligned}
$$

and

$$
\begin{aligned}
& \left(\mathbb{A}^{r}\right)_{2 \gamma_{2} \cdots \gamma_{m}} \\
& \quad=\sum_{i_{2}, i_{3}, \ldots, i_{m}=1}^{n} a_{2 i_{2} i_{3} \cdots i_{m}}\left(\mathbb{A}^{r-1}\right)_{i_{2} \gamma_{2}} \cdots\left(\mathbb{A}^{r-1}\right)_{i_{m} \gamma_{m}}=0 .
\end{aligned}
$$

By (23) and (24), we obtain that there exists at least one zero element in each slice of $\mathbb{A}^{r}$, and thus we complete the proof.

Now we give the characterization of the strongly primitive tensor with order $m$ and dimension 2 .

Theorem 32. Let $\mathbb{A}=\left(a_{i_{1} i_{2} \ldots i_{m}}\right)_{1 \leq i_{j} \leq 2(j=1, \ldots, m)}$ be a nonnegative tensor with order $m$ and dimension $n=2$. Then

(1) A is strongly primitive if and only if one of the following holds:

(a) $\mathbb{A}=\mathbb{J}$ 
(b) $\mathbb{A} \neq \mathbb{J}$ and $a_{1 i_{2} \ldots i_{m}}=a_{211 \ldots 1}=1\left(1 \leq i_{j} \leq 2, j=\right.$ $2, \ldots, m)$;

(c) $\mathbb{A} \neq \mathbb{J}$ and $a_{2 i_{2} \ldots i_{m}}=a_{122 \ldots 2}=1\left(1 \leq i_{j} \leq 2, j=\right.$ $2, \ldots, m)$

(2) if $\mathbb{A}$ is strongly primitive, then $\eta(\mathbb{A}) \leq 2$.

Proof. Firstly, we show that (1) is sufficient. It is easy to see that $\mathbb{A}=\mathbb{J}$ is strongly primitive with $\eta(\mathbb{J})=1$, and if $\mathbb{A}$ satisfies (b) or (c), $\mathbb{A}$ is strongly primitive with $\eta(\mathbb{A})=2$ by Proposition 19 immediately.

Now we show the necessity of (1); that is, if $\mathbb{A}$ does not satisfy the conditions of (a), (b), or (c), then we will show that $\mathbb{A}$ is not strongly primitive. We complete the proof by the following three cases.

Case 1. $a_{1 \alpha}=1$ for any $\alpha \in[2]^{m-1}$ and $a_{211 \ldots 1}=0$.

It is not difficult to find that $M(\mathbb{A})=\left(\begin{array}{ll}1 & 1 \\ 0 & *\end{array}\right)$. Then $\mathbb{A}$ is not primitive by Theorem 26 , and thus $\mathbb{A}$ is not strongly primitive.

Case 2. $a_{2 \alpha}=1$ for any $\alpha \in[2]^{m-1}$ and $a_{122 \ldots 2}=0$.

Similarly, we can find that $M(\mathbb{A})=\left(\begin{array}{ll}* & 0 \\ 1 & 1\end{array}\right)$. Then $\mathbb{A}$ is not primitive by Theorem 26, and thus $\mathbb{A}$ is not strongly primitive.

Case 3. There is at least one zero element in each slice of A.

Then there exist $\alpha_{1}=j_{2} j_{3} \ldots j_{m} \in[2]^{m-1}$ and $\alpha_{2}=$ $k_{2} k_{3} \ldots k_{m} \in[2]^{m-1}$ such that $a_{1 \alpha_{1}}=a_{2 \alpha_{2}}=0$. Thus $\mathbb{A}$ is not strongly primitive by Theorem 31 .

(2) If $\mathbb{A}$ is strongly primitive, by Definition 11 and the proof of (1), we obtain $\eta(\mathbb{A}) \leq 2$ immediately.

Remark 33. By Theorem 32, we can see that the strongly primitive degree $\eta(\mathbb{A})$ of an nonnegative tensor with order $m$ and dimension $n=2$ is irrelevant to its order $m$.

\section{Some Properties and Problems of Order $m$ Dimension $n(\geq 3)$ Strongly Primitive Tensors}

In this section, we will study some properties of the strongly primitive tensors with order $m$ and dimension $n \geq 3$ and propose some questions for further research.

Proposition 34. Let $\mathbb{A}=\left(a_{i_{1} i_{2} \ldots i_{m}}\right)_{1 \leq i_{j} \leq n(j=1, \ldots, m)}$ be a nonnegative tensor with order $m$ and dimension $n$. Let $s \in[n]$, $2 \leq t \leq m, j_{t}^{(s)} \in[n]$, and $\alpha_{s}=j_{2}^{(s)} j_{3}^{(s)} \ldots j_{m}^{(s)}$. If $a_{i \alpha_{s}}=0$ for any $i \in[n]$ and any $s \in[n] \backslash\{i\}$, then there exist $\gamma_{1}, \gamma_{2}, \ldots, \gamma_{n} \in$ $[n]^{(m-1)^{2}}$ such that $\left(\mathbb{A}^{2}\right)_{i \gamma_{k}}=0$ for any $i \in[n]$ and any $k \in$ $[n] \backslash\{i\}$.

Proof. For each $i \in[n]$, let $\beta_{t}^{(k)}=\alpha_{j_{t}^{(k)}} \in[n]^{m-1}$ for any $k \in[n]$ and $2 \leq t \leq m$; then $\overline{\bar{j}_{t}^{(k)} \beta_{t}^{(k)}}=0$ for any $\overline{j_{t}^{(k)}} \in[n] \backslash\left\{j_{t}^{(k)}\right\}$ by $a_{i \alpha_{s}}=0$ for any $i \in[n]$ and any $s \in[n] \backslash\{i\}$. Let $\gamma_{k}=$ $\beta_{2}^{(k)} \beta_{3}^{(k)} \ldots \beta_{m}^{(k)} \in[n]^{(m-1)^{2}}$ for any $k \in[n]$. Now we show $\left(\mathbb{A}^{2}\right)_{i \gamma_{k}}=0$ for any $k \in[n] \backslash\{i\}$.

$$
\begin{aligned}
& \left(\mathbb{A}^{2}\right)_{i \gamma_{k}}=\left(\mathbb{A}^{2}\right)_{i \beta_{2}^{(k)} \beta_{3}^{(k) \ldots} \beta_{m}^{(k)}} \\
& =\sum_{i_{2}, i_{3}, \ldots, i_{m}=1}^{n} a_{i i_{2} i_{3} \cdots i_{m}} a_{i_{2} \beta_{2}^{(k)}} \cdots a_{i_{m} \beta_{m}^{(k)}} \\
& =\sum_{i_{3}, \ldots, i_{m}=1}^{n} a_{i 1 i_{3} \cdots i_{m}} a_{1 \beta_{2}^{(k)}} a_{i_{3} \beta_{3}^{(k)}} \cdots a_{i_{m} \beta_{m}^{(k)}}+\cdots \\
& +\sum_{i_{3}, \ldots, i_{m}=1}^{n} a_{i n i_{3} \cdots i_{m}} a_{n \beta_{2}^{(k)}} a_{i_{3} \beta_{3}^{(k)}} \cdots a_{i_{m} \beta_{m}^{(k)}} \\
& \underline{\underline{a_{j_{2}^{(k)} \beta_{2}^{(k)}}=0}} \sum_{i_{3}, \ldots, i_{m}=1}^{n} a_{i j_{2}^{(k)} i_{3} \cdots i_{m}} a_{j_{2}^{(k)} \beta_{2}^{(k)}} a_{i_{3} \beta_{3}^{(k)}} \cdots a_{i_{m} \beta_{m}^{(k)}} \\
& =\sum_{i_{4}, \ldots, i_{m}=1}^{n} a_{i j_{2}^{(k)} 1 i_{4} \cdots i_{m}} a_{j_{2}^{(k)} \beta_{2}^{(k)}} a_{1 \beta_{3}^{(k)}} a_{i_{4} \beta_{4}^{(k)}} \\
& \cdots a_{i_{m} \beta_{m}^{(k)}}+\cdots+\sum_{i_{4}, \ldots, i_{m}=1}^{n} a_{i j_{2}^{(k)} n i_{4} \cdots i_{m}} a_{j_{2}^{(k)} \beta_{2}^{(k)}} a_{n \beta_{3}^{(k)}} a_{i_{4} \beta_{4}^{(k)}} \\
& \cdots a_{i_{m} \beta_{m}^{(k)}} \\
& \stackrel{\overline{\bar{j}_{3}^{(k)} \beta_{3}^{(k)}}=0}{=} \sum_{i_{4}, \ldots, i_{m}=1}^{n} a_{i j_{2}^{(k)} j_{3}^{(k)} i_{4} \cdots i_{m}} a_{j_{2}^{(k)} \beta_{2}^{(k)}} a_{j_{3}^{(k)} \beta_{3}^{(k)}} a_{i_{4} \beta_{4}^{(k)}} \\
& \cdots a_{i_{m} \beta_{m}^{(k)}}=\cdots \\
& \overline{=} \sum_{i_{m}=1}^{n} a_{i j_{2}^{(k)} j_{3}^{(k)} \ldots j_{m-1}^{(k)} i_{m}} a_{j_{2}^{(k)}} \beta_{2}^{(k)} a_{j_{3}^{(k)} \beta_{3}^{(k)}} \\
& \cdots a_{j_{m-1}^{(k)} \beta_{m-1}^{(k)}} a_{i_{m} \beta_{m}^{(k)}} \\
& =a_{i j_{2}^{(k)} j_{3}^{(k)} \ldots j_{m-1}^{(k)} 1} a_{j_{2}^{(k)} \beta_{2}^{(k)}} a_{j_{3}^{(k)} \beta_{3}^{(k)}} \\
& \cdots a_{j_{m-1}^{(k)} \beta_{m-1}^{(k)}} a_{1 \beta_{m}^{(k)}}+\cdots+a_{i j_{2}^{(k)} j_{3}^{(k)} \ldots j_{m-1}^{(k)} n} a_{j_{2}^{(k)} \beta_{2}^{(k)}} a_{j_{3}^{(k)} \beta_{3}^{(k)}} \\
& \cdots a_{j_{m-1}^{(k)} \beta_{m-1}^{(k)}} a_{n \beta_{m}^{(k)}} \\
& \stackrel{a_{\overline{j_{m}^{(k)}} \beta_{m}^{(k)}}=0}{=} a_{i j_{2}^{(k)} j_{3}^{(k)} \ldots j_{m-1}^{(k)} j_{m}^{(k)}} a_{j_{2}^{(k)} \beta_{2}^{(k)}} a_{j_{3}^{(k)} \beta_{3}^{(k)}} \\
& \cdots a_{j_{m-1}^{(k)} \beta_{m-1}^{(k)}} a_{j_{m}^{(k)} \beta_{m}^{(k)}}=a_{i \alpha_{k}} a_{j_{2}^{(k)} \beta_{2}^{(k)}} a_{j_{3}^{(k)} \beta_{3}^{(k)}} \\
& \cdots a_{j_{m-1}^{(k)} \beta_{m-1}^{(k)}} a_{j_{m}^{(k)} \beta_{m}^{(k)}} \stackrel{a_{i \alpha_{k}}=0}{=} 0 .
\end{aligned}
$$

We note that $k \in[n] \backslash\{i\}$ which means there are $n-1$ zero elements in $i$-th slice of $\mathbb{A}^{2}$; thus we complete the proof by $i \in[n]$.

We note that Proposition 34 is the generalization of Lemma 30; now we will obtain the generalization of Theorem 31. 
Theorem 35. Let $\mathbb{A}=\left(a_{i_{1} i_{2} \ldots i_{m}}\right)_{1 \leq i_{j} \leq n(j=1, \ldots, m)}$ be a nonnegative tensor with order $m$ and dimension $n$. Let $s \in[n], 2 \leq t \leq m$, $j_{t}^{(s)} \in[n]$, and $\alpha_{s}=j_{2}^{(s)} j_{3}^{(s)} \ldots j_{m}^{(s)}$. If $a_{i \alpha_{s}}=0$ for any $i \in[n]$ and any $s \in[n] \backslash\{i\}$, then $\mathbb{A}$ is not strongly primitive.

Proof. Now we show that there exist $\varepsilon_{1}, \varepsilon_{2}, \ldots, \varepsilon_{n} \in[n]^{(m-1)^{r}}$ such that $\left(\mathbb{A}^{r}\right)_{i \varepsilon_{k}}=0$ for any $i \in[n]$ and any $k \in[n] \backslash\{i\}$ by induction on $r(\geq 2)$; say, there exist at least $n-1$ zero elements in each slice of $\mathbb{A}^{r}$ and thus $\mathbb{A}$ is not strongly primitive.

Firstly, by Proposition 34, we know there exist $\gamma_{1}, \gamma_{2}$, $\ldots, \gamma_{n} \in[n]^{(m-1)^{2}}$ such that $\left(\mathbb{A}^{2}\right)_{i \gamma_{k}}=0$ for any $i \in[n]$ and any $k \in[n] \backslash\{i\}$; say, there exist at least $n-1$ zero elements in each slice of $\mathbb{A}^{2}$. Now we assume that there exist $\delta_{1}, \delta_{2}, \ldots, \delta_{n} \in$ $[n]^{(m-1)^{r-1}}$ such that $\left(\mathbb{A}^{r-1}\right)_{i \delta_{k}}=0$ for any $i \in[n]$ and any $k \in[n] \backslash\{i\}$; say, there exist at least $n-1$ zero elements in each slice of $\mathbb{A}^{r-1}$.

Let $\eta_{t}^{(s)}=\delta_{j_{t}^{(s)}}$ for any $s \in[n]$ and $2 \leq t \leq m$; then $\left(\mathbb{A}^{r-1}\right) \bar{j}_{t}^{(s)} \eta_{t}^{(s)}=0$ for any $\overline{j_{t}^{(s)}} \in[n] \backslash\left\{j_{t}^{(s)}\right\}$ by $\left(\mathbb{A}^{r-1}\right)_{i \delta_{k}}=0$ for any $i \in[n]$ and any $k \in[n] \backslash\{i\}$. Let $\varepsilon_{k}=\eta_{2}^{(k)} \eta_{3}^{(k)} \ldots \eta_{m}^{(k)} \in$ $[n]^{(m-1)^{r}}$ for any $k \in[n]$. Now we show $\left(\mathbb{A}^{r}\right)_{i \varepsilon_{k}}=0$ for any $i \in[n]$ and any $k \in[n] \backslash\{i\}$.

By (7) and similar proof of Proposition 34, we have

$$
\begin{aligned}
\left(\mathbb{A}^{r}\right)_{i \varepsilon_{k}} & =\left(\mathbb{A}^{r}\right)_{i \eta_{2}^{(k)} \eta_{3}^{(k)} \cdots \eta_{m}^{(k)}} \\
& =\sum_{i_{2}, i_{3}, \ldots, i_{m}=1}^{n} a_{i i_{2} i_{3} \cdots i_{m}}\left(\mathbb{A}^{r-1}\right)_{i_{2} \eta_{2}^{(k)}} \cdots\left(\mathbb{A}^{r-1}\right)_{i_{m} \eta_{m}^{(k)}} \\
& =0
\end{aligned}
$$

and then we complete the proof.

Proposition 36. Let $\mathbb{A}=\left(a_{i_{1} i_{2} \cdots i_{m}}\right)_{1 \leq i_{j} \leq n(j=1, \cdots, m)}$ be a nonnegative tensor with order $m$ and dimension $n$, and $M(\mathbb{A})$ be the majorization matrix of $\mathbb{A}$. If there exist $i, j \in[n]$, such that $(M(\mathbb{A}))_{i j}>0,(M(\mathbb{A}))_{u j}=0$ for any $u \in[n] \backslash\{i\}$ and $(M(\mathbb{A}))_{j i}>0,(M(\mathbb{A}))_{v i}=0$ for any $v \in[n] \backslash\{j\}$, then $\mathbb{A}$ is not primitive, and thus $\mathbb{A}$ is not strongly primitive.

Proof. Firstly, we show the following assertion:

If $k$ is odd, then $\left(M\left(\mathbb{A}^{k}\right)\right)_{i j}>0,\left(M\left(\mathbb{A}^{k}\right)\right)_{j i}>0$, $\left(M\left(\mathbb{A}^{k}\right)\right)_{u j}=0$ for any $u \in[n] \backslash\{i\},\left(M\left(\mathbb{A}^{k}\right)\right)_{v i}=0$ for any $v \in[n] \backslash\{j\}$.

If $k$ is even, then $\left(M\left(\mathbb{A}^{k}\right)\right)_{i i}>0,\left(M\left(\mathbb{A}^{k}\right)\right)_{j j}>0$, $\left(M\left(\mathbb{A}^{k}\right)\right)_{u i}=0$ for any $u \in[n] \backslash\{i\},\left(M\left(\mathbb{A}^{k}\right)\right)_{v j}=0$ for any $v \in[n] \backslash\{j\}$.

When $k=1$, the above result holds which is obvious. When $k=2$, by Definition 7 and (7), we have

$$
\begin{aligned}
\left(M\left(\mathbb{A}^{2}\right)\right)_{i i} & =\left(\mathbb{A}^{2}\right)_{i i \cdots i} \\
& =\sum_{i_{2}, i_{3}, \ldots, i_{m}=1}^{n} a_{i i_{2} i_{3} \cdots i_{m}} a_{i_{2} i \cdots i} \cdots a_{i_{m} i \cdots i} \\
& =a_{i j \cdots j}\left(a_{j i \cdots i}\right)^{m-1} \\
& =(M(\mathbb{A}))_{i j}\left((M(\mathbb{A}))_{j i}\right)^{m-1}>0,
\end{aligned}
$$

and for any $u \in[n] \backslash\{i\}$, we have

$$
\begin{aligned}
\left(M\left(\mathbb{A}^{2}\right)\right)_{u i} & =\left(\mathbb{A}^{2}\right)_{u i \cdots i} \\
& =\sum_{i_{2}, i_{3}, \ldots, i_{m}=1}^{n} a_{u i_{2} i_{3} \cdots i_{m}} a_{i_{2} i \cdots i} \cdots a_{i_{m} i \cdots i} \\
& =a_{u j \cdots j}\left(a_{j i \cdots i}\right)^{m-1} \\
& =(M(\mathbb{A}))_{u j}\left((M(\mathbb{A}))_{j i}\right)^{m-1}=0 .
\end{aligned}
$$

Similarly, we can show $\left(M\left(\mathbb{A}^{2}\right)\right)_{j j}>0$ and $\left(M\left(\mathbb{A}^{2}\right)\right)_{v j}=0$ for any $v \in[n] \backslash\{j\}$.

Now we assume that, for any $k$, the above assertion holds. Then, for $k+1$, we consider the following two cases.

Case $1 . k$ is odd.

Then, by (7), we have

$$
\begin{aligned}
& \left(M\left(\mathbb{A}^{k+1}\right)\right)_{i i}=\left(\mathbb{A}^{k+1}\right)_{i i \cdots i} \\
& \quad=\sum_{i_{2}, i_{3}, \ldots, i_{m}=1}^{n} a_{i i_{2} i_{3} \cdots i_{m}}\left(\mathbb{A}^{k}\right)_{i_{2} i \cdots i} \cdots\left(\mathbb{A}^{k}\right)_{i_{m} i \cdots i} \\
& =a_{i j \cdots j}\left(\left(\mathbb{A}^{k}\right)_{j i \cdots i}\right)^{m-1} \\
& =(M(\mathbb{A}))_{i j}\left(\left(M\left(\mathbb{A}^{k}\right)\right)_{j i}\right)^{m-1}>0,
\end{aligned}
$$

and, for any $u \in[n] \backslash\{i\}$, we have

$$
\begin{aligned}
& \left(M\left(\mathbb{A}^{k+1}\right)\right)_{u i}=\left(\mathbb{A}^{k+1}\right)_{u i \cdots i} \\
& =\sum_{i_{2}, i_{3}, \ldots, i_{m}=1}^{n} a_{u i_{2} i_{3} \cdots i_{m}}\left(\mathbb{A}^{k}\right)_{i_{2} i \cdots i} \cdots\left(\mathbb{A}^{k}\right)_{i_{m} i \cdots i} \\
& =a_{u j \cdots j}\left(\left(\mathbb{A}^{k}\right)_{j i \cdots i}\right)^{m-1} \\
& =(M(\mathbb{A}))_{u j}\left(\left(M\left(\mathbb{A}^{k}\right)\right)_{j i}\right)^{m-1}=0 .
\end{aligned}
$$

Similarly, we can show $\left(M\left(\mathbb{A}^{k+1}\right)\right)_{j j}>0$ and $\left(M\left(\mathbb{A}^{k+1}\right)\right)_{v j}=0$ for any $v \in[n] \backslash\{j\}$.

Case 2. $k$ is even.

By (7) and similar proof of Case 1, we can show $\left(M\left(\mathbb{A}^{k+1}\right)\right)_{i j}>0,\left(M\left(\mathbb{A}^{k+1}\right)\right)_{j i}>0,\left(M\left(\mathbb{A}^{k+1}\right)\right)_{u j}=0$ for any $u \in[n] \backslash\{i\}$, and $\left(M\left(\mathbb{A}^{k+1}\right)\right)_{v i}=0$ for any $v \in[n] \backslash\{j\}$.

By Proposition 8 and the above assertion, we know $\mathbb{A}$ is not primitive, and thus $\mathbb{A}$ is not strongly primitive.

Let $\mathbb{A}=\left(a_{i_{1} i_{2} \cdots i_{m}}\right)_{1 \leq i_{j} \leq n(j=1, \cdots, m)}$ be a nonnegative strongly primitive tensor with order $m$ and dimension $n$. When $n=$ 2 , we know $\eta(\mathbb{A}) \leq 2$ by Theorem 32. When $n \geq 3$, we do not know the value or bound of $\eta(\mathbb{A})$. Even $n=3$, we do not find out all strongly primitive tensors. Thus we think it is not easy to obtain the value or bound of $\eta(\mathbb{A})$. Based on the computation of the case $n=3$, we propose the following problem for further research. 
Question 37. Let $n \geq 3$, $\mathbb{A}=\left(a_{i_{1} i_{2} \cdots i_{m}}\right)_{1 \leq i_{j} \leq n(j=1, \cdots, m)}$ be a nonnegative strongly primitive tensor with order $m$ and dimension $n$. Then $\eta(\mathbb{A})<(n-1)^{2}+1$.

In $[7,9]$, the authors gave some algebraic characterizations of a nonnegative primitive tensor, and in [11] the authors showed that a nonnegative tensor is primitive if and only if the greatest common divisor of all the cycles in the associated directed hypergraph is equal to 1 . It is natural for us to consider the following.

Question 38. Study the algebraic or graphic characterization of a nonnegative strongly primitive tensor.

We are sure the above two questions are interesting and not easy.

\section{Data Availability}

No data were used to support this study.

\section{Conflicts of Interest}

The authors declare that they have no conflicts of interest.

\section{Acknowledgments}

L. You's research is supported by the National Natural Science Foundation of China (Grant no. 11571123) and the Guangdong Provincial Natural Science Foundation (Grant no. 2015A030313377). P. Yuan's research is supported by the NSF of China (Grant no. 11671153). Y. Chen's research is supported by the Scientific Research Foundation of Graduate School of South China Normal University (Grant no. 2015lkxm19).

\section{References}

[1] L. Qi, "Eigenvalues of a real supersymmetric tensor," Journal of Symbolic Computation, vol. 40, no. 6, pp. 1302-1324, 2005.

[2] L.-H. Lim, "Singular values and eigenvalues of tensors: a variational approach," in Proceedings of the 1st IEEE International Workshop on Computational Advances in Multi-Sensor Adaptive Processing (CAMSAP '05), pp. 129-132, Puerto Vallarta, Mexico, December 2005.

[3] N. J. Pullman, Matrix theory and its applications, Selected topics, Pure and Applied Mathematics 35, Marcel Dekker, Inc., New York, USA, 1976.

[4] K.-C. Chang, K. J. Pearson, and T. Zhang, "Primitivity, the convergence of the NQZ method, and the largest eigenvalue for nonnegative tensors," SIAM Journal on Matrix Analysis and Applications, vol. 32, no. 3, pp. 806-819, 2011.

[5] J.-Y. Shao, "A general product of tensors with applications," Linear Algebra and Its Applications, vol. 439, no. 8, pp. 23502366, 2013.

[6] K. Pearson, "Essentially positive tensors," International Journal of Algebra, vol. 4, pp. 421-427, 2010.

[7] P. Yuan, Z. He, and L. You, "A conjecture on the primitive degree of tensors," Linear Algebra and Its Applications, vol. 450, pp. 175$185,2014$.
[8] P. Yuan, Z. He, and L. You, "Further results and some open problems on the primitive degree of nonnegative tensors," Linear Algebra and Its Applications, vol. 480, pp. 72-92, 2015.

[9] Z. He, P. Yuan, and L. You, "On the exponent set of nonnegative primitive tensors," Linear Algebra and Its Applications, vol. 465, pp. 376-390, 2015.

[10] J.-Y. Shao, H.-Y. Shan, and L. Zhang, "On some properties of the determinants of tensors," Linear Algebra and Its Applications, vol. 439, no. 10, pp. 3057-3069, 2013.

[11] L.-B. Cui, W. Li, and M. K. Ng, "Primitive tensors and directed hypergraphs," Linear Algebra and Its Applications, vol. 471, pp. 96-108, 2015. 


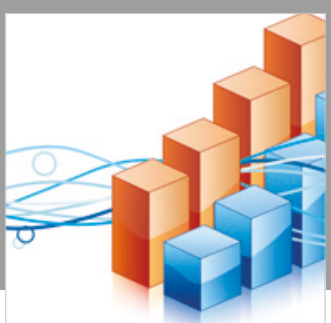

Advances in

Operations Research

\section{-n-m}
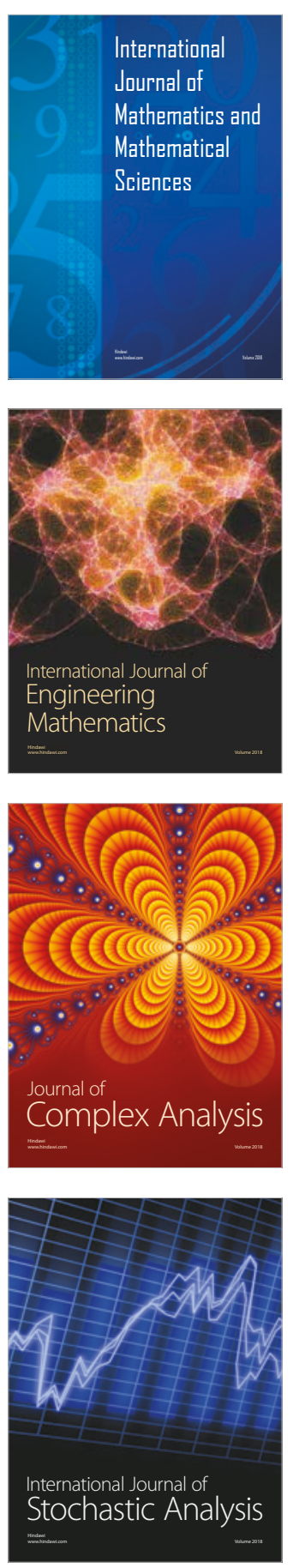
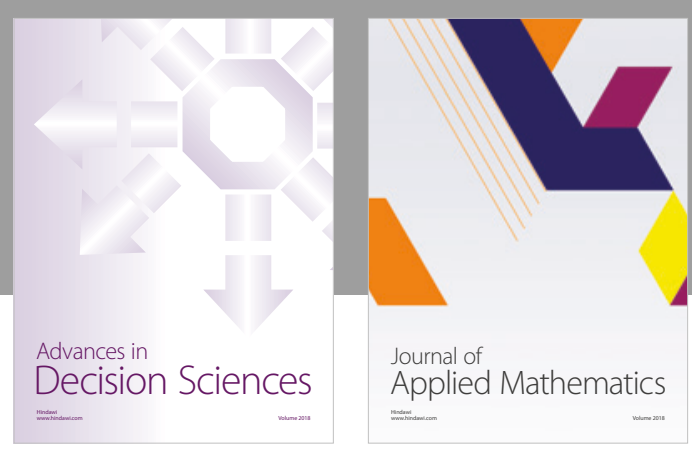

Journal of

Applied Mathematics
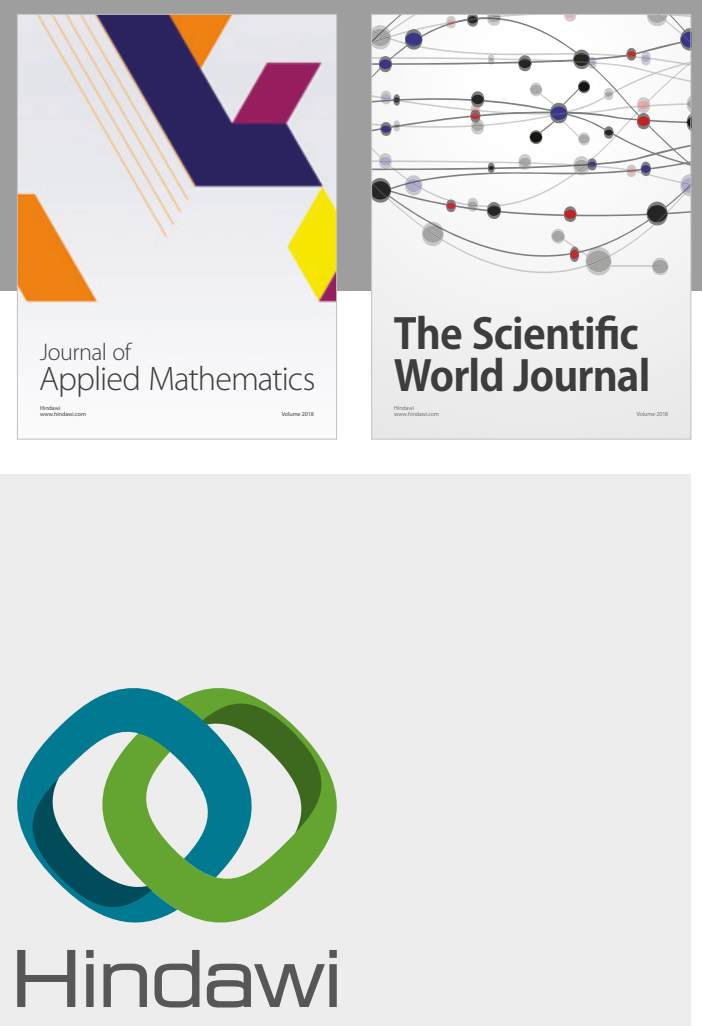

Submit your manuscripts at

www.hindawi.com

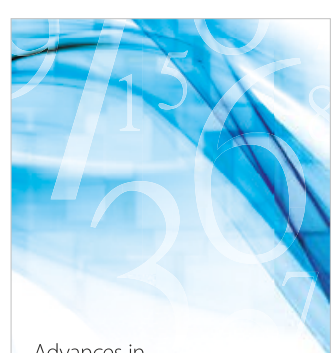

Advances in
Numerical Analysis
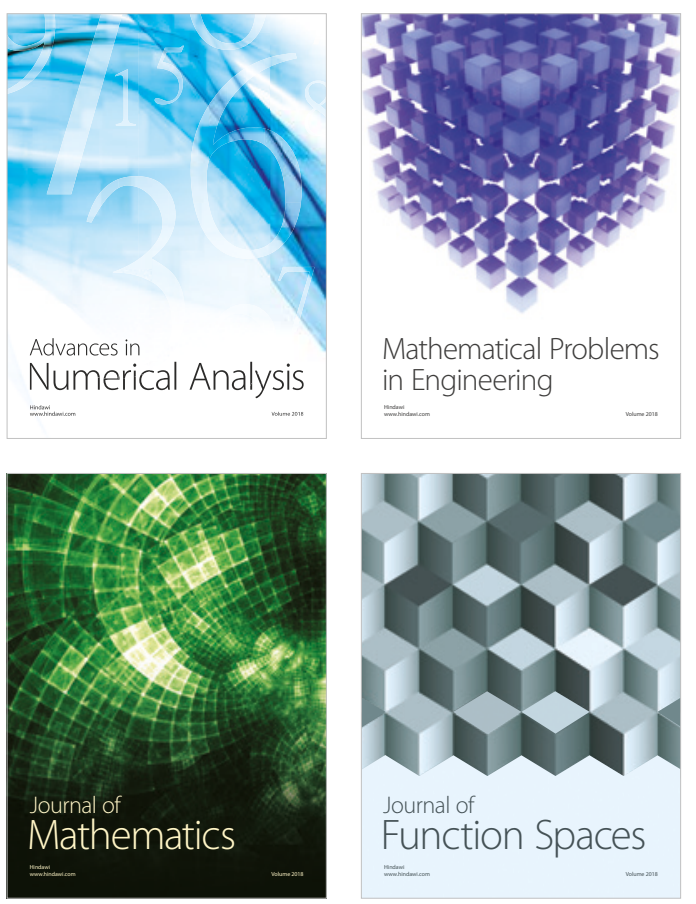

Mathematical Problems in Engineering

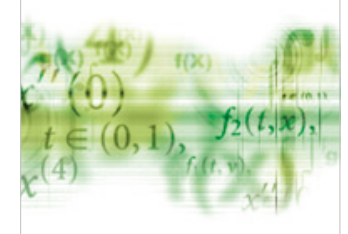

International Journal of

Differential Equations

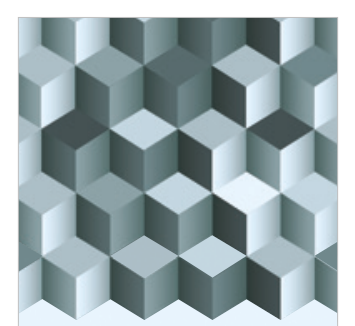

Journal of

Function Spaces

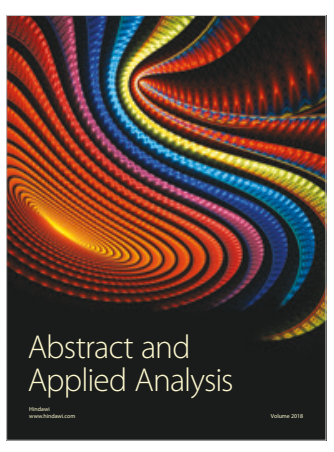

The Scientific

World Journal

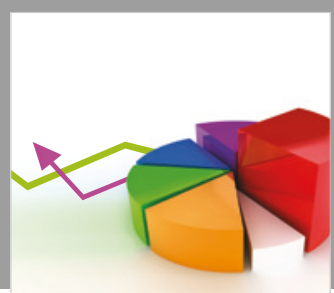

Journal of

Probability and Statistics
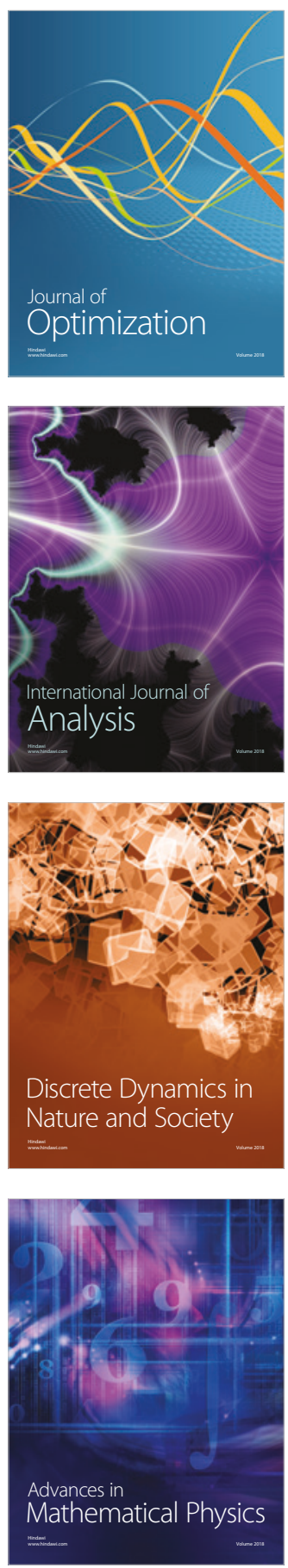